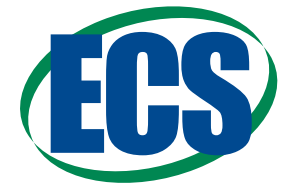

Jes focus Issue on Advances in Modern Polymer Electrolyte Fuel Cells in Honor of Shimshon Gottesfeld

\title{
Spatially Resolved Quantification of Ionomer Degradation in Fuel Cells by Confocal Raman Microscopy
}

\author{
Thomas Böhm, ${ }^{1}$ Riko Moroni, ${ }^{1}$ Matthias Breitwieser, ${ }^{1,2}$ Simon Thiele, ${ }^{3,4}$ \\ and Severin Vierrath $1,2, *, \mathrm{z}$ \\ ${ }^{1}$ Laboratory for MEMS Applications, IMTEK Department of Microsystems Engineering, University of Freiburg, 79110 \\ Freiburg, Germany \\ ${ }^{2}$ Hahn-Schickard, 79110 Freiburg, Germany \\ ${ }^{3}$ Forschungszentrum Jülich GmbH, Helmholtz-Institute Erlangen-Nürnberg for Renewable Energy (IEK-11), 91058 \\ Erlangen, Germany \\ ${ }^{4}$ Department of Chemical and Biological Engineering, Friedrich-Alexander-Universität Erlangen-Nürnberg, 91058 \\ Erlangen, Germany
}

\begin{abstract}
Ionomer membranes are crucial components of many electrochemical devices. In this work, confocal Raman microscopy is employed to characterize Nafion ionomers quantitatively in pristine status and after usage as a proton exchange membrane in a fuel cell. Confocal Raman microscopy allows non-destructive thickness and equivalent weight measurements of Nafion with a 95\% confidence interval of $\pm 13 \mathrm{~g} \mathrm{~mol}^{-1}$ at an equivalent weight of $1000 \mathrm{~g} \mathrm{~mol}^{-1}$, which is significantly more accurate than previously reported methods. Characterization can be performed at a spatial resolution better than $2 \mu \mathrm{m}$, providing insights into local membrane degradation after fuel cell operation. Membrane thinning to less than $40 \%$ of the initial thickness of Nafion NR-211 occurs after a $100 \mathrm{~h}$ open circuit voltage hold, accompanied by an anisotropic increase of the equivalent weight from $1035 \mathrm{~g} \mathrm{~mol}^{-1}$ to an average of $1200 \mathrm{~g}$ $\mathrm{mol}^{-1}$. Most pronounced increases are found close to the anode. Further, the characterization of a Nafion XL membrane shows that its microporous reinforcement is represented as increased equivalent weight with local heterogeneities within the membrane. These results show that confocal Raman microscopy is a valuable tool to investigate ionomers that are used as ion exchange membranes in electrochemical devices.

(C) The Author(s) 2019. Published by ECS. This is an open access article distributed under the terms of the Creative Commons Attribution 4.0 License (CC BY, http://creativecommons.org/licenses/by/4.0/), which permits unrestricted reuse of the work in any medium, provided the original work is properly cited. [DOI: 10.1149/2.0051907jes]

(cc) BY
\end{abstract}

Manuscript submitted November 9, 2018; revised manuscript received January 11, 2019. Published March 2, 2019. This paper is part of the JES Focus Issue on Advances in Modern Polymer Electrolyte Fuel Cells in Honor of Shimshon Gottesfeld.

The ionomer membrane is crucial for the performance and safety of electrochemical devices such as proton exchange membrane fuel cells (PEMFC). For its function as an electrolyte between anode and cathode, the membrane needs to have high proton conductivity and therefore should be as thin as possible, while it is also required to be chemically and thermally stable. At the same time the ionomer needs to be mechanically stable to prevent gas crossover and electrical shorting between the two electrodes. ${ }^{1}$

For decades, the 'gold-standard' ionomers are perfluorosulfonated acids (PFSA), with Nafion as the most prominent example. Nafion consists of a hydrophobic backbone composed of polytetrafluoroethylene (PTFE) with hydrophilic sidechains that carry a sulfonic acid end group and thereby allow the polymer to conduct protons. ${ }^{2}$ Although PFSA membranes are chemically and mechanically very stable, they still suffer from degradation over time due to the harsh working conditions in a fuel cell: during operation and cycling, the membrane is exposed to temperature and humidity changes, high electrochemical potentials, acidic environments, and mechanical stress.

Membrane degradation in a PEMFC leads to membrane thinning, the formation of pinholes, increased gas cross-over, and electrical shorts. ${ }^{3}$ The underlying mechanisms can generally be divided into mechanical, thermal, and chemical membrane degradation. ${ }^{4} \mathrm{Mem}-$ brane defects from the manufacturing process, incorrect fuel cell assembly, or inaccuracies in the structure of electrodes or flow fields may lead to unbalanced local mechanical stress on the membrane. In addition, when the fuel cell is operating, the membrane experiences further mechanical stress due to membrane swelling and shrinking, caused by changing membrane water content upon dynamic operation. Membrane integrity can be compromised by this mechanical stress, which results in a reduced contact area between membrane and electrodes, and the direct reaction of $\mathrm{H}_{2}$ and $\mathrm{O}_{2}$ by gas crossover, adding heat locally and thereby stressing the membrane thermally. ${ }^{4,5}$ In addition to thermal stress, gas crossover supports undesired side reactions

*Electrochemical Society Student Member.

${ }^{\mathrm{z}}$ E-mail: severin.vierrath@imtek.uni-freiburg.de at the electrodes that accelerate chemical membrane degradation. ${ }^{5}$ Although the PTFE-backbone of PFSA-membranes like Nafion is thermally stable up to $400^{\circ} \mathrm{C},{ }^{6}$ ionomer membranes can be damaged already at much lower temperatures. While the polymer decomposition starts at around $280^{\circ} \mathrm{C},{ }^{6}$ the $\alpha$-relaxation of Nafion, which significantly increases molecular mobility, already occurs at

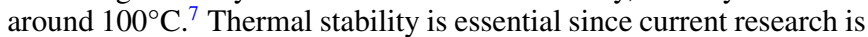
focusing on medium-temperature PEMFC operation toward $120^{\circ} \mathrm{C}$, which has several advantages such as increased catalytic activity, high resistance toward CO poisoning, and easy cooling in automotive applications. ${ }^{8}$ The third mechanism of membrane degradation is a chemical decomposition of the ionomer. Especially at high potential and low humidity conditions this type of membrane destruction propagates rapidly ${ }^{9}$ by radicals attacking backbone end groups as well as sidechains of the ionomer membrane. ${ }^{10}$ Furthermore, chemical and mechanical membrane degradation mechanisms are highly related since chemical membrane destruction leads to membrane thinning and therefore supports the formation of pinholes in the membrane. ${ }^{11}$

Chemical membrane degradation occurs in the presence of radicals. Radical formation takes place at the anode and the cathode of a fuel cell in the presence of both $\mathrm{H}_{2}$ and $\mathrm{O}_{2}$, which form $\mathrm{H}_{2} \mathrm{O}_{2}$ and subsequently radicals. For fuel cells it has been proposed that $\mathrm{H}_{2} \mathrm{O}_{2}$ is preferentially produced on the anode side. ${ }^{12}$ However, because $\mathrm{H}_{2} \mathrm{O}_{2}$ diffuses through the membrane, ${ }^{13} \mathrm{H}_{2} \mathrm{O}_{2}$ formation and membrane degradation are not necessarily spatially linked. ${ }^{5}$ It has been suggested that the decomposition of $\mathrm{H}_{2} \mathrm{O}_{2}$ into highly reactive $\bullet \mathrm{OH}$ and $\bullet \cdot \mathrm{OOH}$ occurs at the Pt catalyst of the electrodes ${ }^{13}$ and/or by cation contamination of the fuel cell (e.g. $\mathrm{Fe}^{2+} / \mathrm{Fe}^{3+}$, which is also used for ex situ membrane degradation studies in the Fenton's test), ${ }^{14}$ that can occur upon migration of cations from fuel cell components into the membrane. ${ }^{15}$ Ohguri et al. found that the formation of $\bullet \mathrm{OH}$ radicals depends on the $\mathrm{Pt}$ loading of the membrane electrode assembly (MEA), ${ }^{16}$ meaning that for example a higher Pt loading of the cathode catalyst layer as compared to the anode may lead to an accelerated membrane degradation at the cathode side. To date there are studies that suggest chemical membrane 
degradation to take place preferentially on the anode side ${ }^{16-18}$ or the cathode side ${ }^{15,19-21}$ of a MEA. Taking the contradictory results from literature into account, a strong influence of the operating conditions (e.g. cell voltage), the MEA components (e.g. Pt loading), and the overall cell design (e.g. material of flow fields) on membrane degradation can be expected. Thus, a reliable experimental approach for membrane characterization independent from cell design is required in order to elucidate chemical degradation modes.

Especially for chemically stabilized Nafion membranes with a reduced number of terminal $-\mathrm{COOH}$ end groups, ${ }^{22}$ chemical degradation is supposed to occur via radicals attacking the sidechains of the PFSA,${ }^{10}$ which reduces the amount of $-\mathrm{SO}_{3}{ }^{-}$groups in the polymer. The amount of $-\mathrm{SO}_{3}{ }^{-}$groups in the polymer is typically quantified in gram of polymer per mole $-\mathrm{SO}_{3}{ }^{-}$groups as the equivalent weight (EW). Thus, the EW is directly related to the stability and crystallinity of the ionomer as well as to its proton conductivity. ${ }^{23}$

A variety of methods have been described that allow EW quantification of an ionomer. The standard method is titrimetry, which requires reliable drying and weight measurement as well as a titration step in order to calculate the EW. ${ }^{24}$ More sophisticated approaches such as elemental analysis, energy dispersive X-ray spectroscopy (EDX), or nuclear magnetic resonance spectroscopy have also been used. ${ }^{25}$ All of these methods can be applied to pure membranes, while reinforced membranes require approaches with spatial resolution. Moukheiber et al. showed that EDX-mapping is a suitable method for the analysis of Nafion XL, a PFSA membrane that is reinforced with a PTFElayer ${ }^{25}$ However, only sample surfaces can be investigated with EDX, requiring cross-sections of membranes for a depth analysis. In addition, EDX-analysis comes along with the typical restrictions of scanning electron microscopy (SEM) including e.g. small sample volumes, and possible charging effects on electrically insulating polymer samples.

Thus, a very promising approach for ionomer characterization is vibrational spectroscopy. This non-destructive analysis method is based on the detection of molecular vibrations, which are very sensitive toward changes in the molecular sample structure. PFSAs have been extensively studied using infrared (IR) and Raman spectroscopy. Besides a basic characterization of the ionomer itself, ${ }^{26}$ Raman and IR spectroscopy have been used to investigate water in Nafion, ${ }^{27,28}$ even within an operating fuel cell. ${ }^{29}$ Also, a qualitative analysis of the sidechainloss of Nafion upon membrane degradation using Raman spectroscopy has been reported. ${ }^{30}$ Additionally, the EW of Nafion has been quantified by IR spectroscopy. ${ }^{31}$ This method is highly related to Raman spectroscopy since they both are based on the detection of molecular vibrations, but they produce different spectra of a sample due to different sensitivities of Raman and IR spectroscopy toward different vibrational modes of molecules. Further, the application options of Raman and IR spectroscopy differ. Raman spectroscopy can be implemented relatively easily in confocal laser scanning microscopes as these devices are already designed for local sample excitation with laser light in the visible spectral range at a high spatial accuracy and resolution. IR spectroscopy, on the other hand, lacks this high spatial resolution due to the diffraction limited resolution of light microscopy because of the long IR wavelengths being used. Raman spectroscopy can be readily performed in aqueous solutions due to the relatively weak and distinct Raman peaks of water in contrast to the massive IR absorption spectrum of water. ${ }^{32}$ Thus, confocal Raman microscopy is advantageous in the (local) analysis of hygroscopic materials or specimens that change their characteristics upon changes in their hydration level. A study by Korzeniewski et al. proved that Raman and IR spectroscopy are capable of a quantitative EW analysis of different ionomers, yet the authors only applied their quantification method on pristine ionomer samples. ${ }^{33}$

In this work, we use the described advantages of confocal Raman microscopy for the first time to quantify the EW of different commercially available pure Nafion membranes as well as of a PTFE-reinforced Nafion XL membrane. To our knowledge, this is the first study to show that this approach is capable of reliable thickness and local EW measurements of ionomer membranes in the pristine state as well as after operation in a fuel cell, allowing determining loss of sidechains and membrane thinning quantitatively.

\section{Experimental}

Nafion membranes and ionomer film preparation.-Nafion dispersions DE2029, D2020 (Ion Power, USA), and D2021 (FuelCellStore, USA) were drop-cast on glass slides and solvents were allowed to evaporate at ambient air conditions. Aquivion D7225BS (Sigma-Aldrich, USA) was prepared equally, however a thermal annealing step was performed in order to render the ionomer film water-insoluble. After drop-casting and solvent evaporation at room temperature, Aquivion D72-25BS was thermally annealed for $2 \mathrm{~h}$ at $180^{\circ} \mathrm{C}$ on a hotplate. Nafion membranes N117, NR-211, NR-212, and Nafion XL (Ion Power, USA) were investigated as received from the manufacturer.

The manufacturer provides an acid capacity range (ion exchange capacity; IEC) in meq $\mathrm{g}^{-1}$ of the ionomers which allows to calculate the $\mathrm{EW}$ in $\mathrm{g} \mathrm{mol}^{-1}$ by $1000 / \mathrm{IEC}=\mathrm{EW}$. The EW of an ionomer is linked to the number of backbone repeat units per sidechain $m$ (compare Figure 1a) by $\mathrm{EW}=m \cdot \mathrm{M}_{\mathrm{bb}}+\mathrm{M}_{\mathrm{sc}} .{ }^{2}$ Note that the backbone repeat unit that carries the sidechain is considered as being part of the sidechain in the conversion from EW to $m$. Thus, backbone and sidechain are labeled accordingly in Figure 1a. For Nafion and Aquivion the molecular mass of the backbone repeat unit $\mathrm{M}_{\mathrm{bb}}$ equals $100 \mathrm{~g} \mathrm{~mol}^{-1}$. The molecular mass of the sidechain $\mathrm{M}_{\mathrm{sc}}$ is $278 \mathrm{~g} \mathrm{~mol}^{-1}$ for Aquivion and $444 \mathrm{~g} \mathrm{~mol}^{-1}$ for Nafion.

Fuel cell testing and accelerated membrane degradation.-To shorten the time of ageing, four $1 \times 1 \mathrm{~cm}^{2}$ MEAs were assembled in one cell fixture with a $25 \mathrm{~cm}^{2}$ serpentine flow field and operated in a Scribner 850e fuel cell test system. The cells were separated by PTFEgaskets $\left(240 \mu \mathrm{m}\right.$ thickness on each side) with cut-outs of $1.1 \times 1.1 \mathrm{~cm}^{2}$ for each cell (see Figure S1). The MEAs were prepared with Nafion NR-211 membranes of $3 \times 3 \mathrm{~cm}^{2}$ and gas diffusion electrodes of $1 \times 1 \mathrm{~cm}^{2}$ with a Pt loading of $0.25 \mathrm{mg} \mathrm{cm}^{-2}$ (cathode) and $0.05 \mathrm{mg}$ $\mathrm{cm}^{-2}$ (anode) on Freudenberg H24C5 (provided by Greenerity, Germany). The cells were equilibrated over $1 \mathrm{~h}$ by $1 \mathrm{~min}$ voltage steps of open circuit voltage (OCV), $0.6 \mathrm{~V}$, and $0.3 \mathrm{~V}$; at full humidification, $80^{\circ} \mathrm{C}$ cell temperature, ambient pressure, and $\mathrm{H}_{2} / \mathrm{O}_{2}$. The accelerated stress test was conducted as OCV hold according to the department of energy (DOE) protocol. ${ }^{34} \mathrm{H}_{2}$ crossover and electrical shorts were measured by linear sweep voltammetry (LSV) under $\mathrm{H}_{2} / \mathrm{N}_{2}$, fully humidified, ambient pressure, and $80^{\circ} \mathrm{C}$ (Figure S2a). The high frequency resistance (HFR) was measured by current interrupt at $3 \mathrm{kHz}$ (Figure S2b). After $25 \mathrm{~h}, 50 \mathrm{~h}$, and $75 \mathrm{~h}$ of OCV hold, one of the MEAs in the cell fixture was removed for Raman measurements and replaced by a $3 \times 3 \mathrm{~cm}^{2}$ polyvinylidene fluoride (PVDF) foil to ensure gas tightness of the setup, followed by continuing the OCV hold. The last MEA was retrieved for Raman investigation after a total OCV hold time of $100 \mathrm{~h}$

MEA preparation for Raman measurements.-Carbon black at the electrodes is highly sensitive toward thermal degradation by laser irradiation and upon decomposition also destroys surrounding ionomer. Thus, all traces of electrode material needed to be removed prior to Raman measurements. A study that investigated ageing in Aquivion membranes by confocal Raman microscopy circumvented this problem by imaging cross sections of the MEA ${ }^{35}$ However, using a single cross section of a MEA limits the accessible cell area to a single line. Further, preparing cross sections by physical sectioning e.g. with a razor blade might leave remnant carbon particles on the area to be investigated, which might impair subsequent Raman analyses. Therefore, we removed the electrodes of MEAs with a pulsed laser, which only ablates the electrodes without harming the membrane. A laser engraver (Trotec Speedy 300, Trotec, Austria) with a pulsed fiber laser $(1064 \mathrm{~nm})$ was used at $5 \mathrm{~W}$, at a pulse frequency of $20 \mathrm{kHz}$, and at a speed of $0.2 \mathrm{~m} \mathrm{~s}^{-1}$ to remove electrodes. The membrane was fixed at the outer edges, with no physical contact to the device's sample 

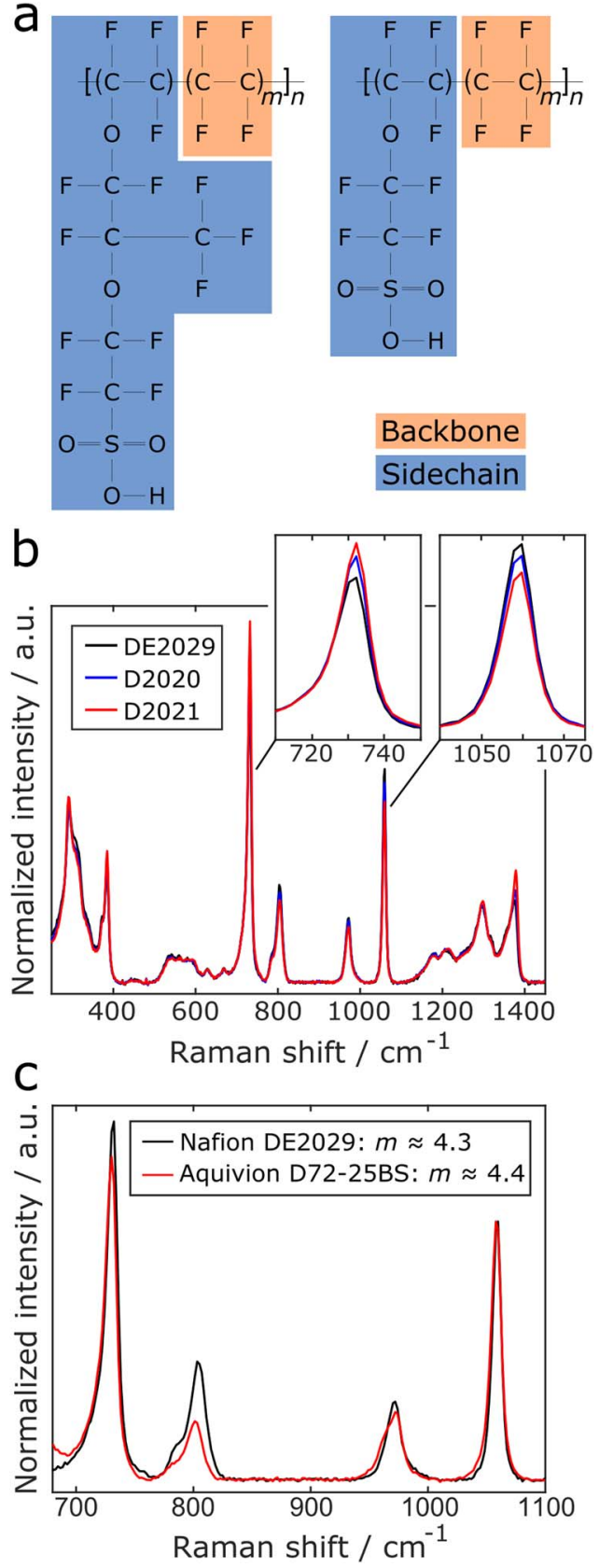

Figure 1. Raman spectra and structure formulas of Nafion and Aquivion. a) Structure formulas of LSC ionomer Nafion (left) and SSC ionomer Aquivion (right). The sidechains of the ionomers are highlighted in blue and the backbone repeat units in orange. $m$ represents the number of backbone repeat units per sidechain and $n$ equals the total repeat number. b) Raman-spectra of Nafion dispersions DE2029, D2020, and D2021 after normalization to the spectral area from 475 to $1500 \mathrm{~cm}^{-1}$. Insets show the $v_{\mathrm{s}}(\mathrm{C}-\mathrm{F})_{\mathrm{bb}}$ and the $\nu_{\mathrm{s}}(\mathrm{S}-\mathrm{O})$ vibrations. c) Comparison of the Raman spectra of Nafion (black) and Aquivion (red). Spectra are normalized to the intensity of the $\nu_{\mathrm{s}}(\mathrm{S}-\mathrm{O})$ mode at $1060 \mathrm{~cm}^{-1}$.

stage below the laser target area. After 20 cycles of laser ablation on the active cell area, the electrodes were removed on both sides of the membrane without visual remnants being left.

In order to confirm that the laser ablation process did not alter the membrane, we compared the EW and thickness of a pristine NR-211 membrane with a Nafion NR-211 membrane that was part of a MEA and used within a break-in procedure. The latter is required to create a membrane with attached electrodes, as it is the case for MEAs after fuel cell operation. No significant differences were noticeable in the characteristics of pristine and electrode ablated membrane (Table S1).
Raman measurements.-When attempting a quantitative analysis of Raman spectra, it is important to keep the measurement conditions as constant as possible. For example, the sulfonyl group peak $v_{\mathrm{s}}(\mathrm{S}-\mathrm{O})$ is sensitive toward a change of the counter-ion. ${ }^{36}$ Another important factor for the intensity of this Raman band is the hydration status of the ionomer. ${ }^{37} \mathrm{~A}$ higher hydration level leads to a higher peak intensity and a slight red-shift of the $\nu_{\mathrm{s}}(\mathrm{S}-\mathrm{O})$ mode (Figure S3a). Thus, the quantitative analysis approach presented in this work can only be applied to ionomers at the same environmental conditions. According to Alberti et al., a more than $180 \mu \mathrm{m}$ thick Nafion membrane (N117) already reaches a hydration level of more than $95 \%$ after immersion in water at room temperature for $1 \mathrm{~h} .{ }^{38}$ Therefore, Raman measurements of ionomers were conducted after at least $1 \mathrm{~h}$ of immersion in DI water. Measurements were performed using a dedicated sample holder (Figure S3b and Figure S3c) for the analysis of hydrated samples and with a water immersion objective (Zeiss W Plan-Apochromat $63 \times / 1.0)$. Additionally, the effect of membrane color changes needs to be taken into account. Nafion membranes turn from transparent to yellowish-brown upon long term storage. The color change is not a chemical reaction of the ionomer but rather occurs due to the uptake of organic compounds from the surrounding atmosphere. ${ }^{39}$ These compounds result in fluorescent background signals in Raman spectra and potentially interfere with the analysis of the spectra. It has been shown that prolonged irradiation of a sample with the Raman excitation laser can effectively reduce background signals in samples exhibiting fluorescence (photo-bleaching). ${ }^{40}$ We adapted this process to our Raman measurements to eliminate possible skewing of Raman peak intensity ratios due to an underlying fluorescence signal. Background fluorescence was removed by photo-bleaching prior to Raman measurements with the same laser intensity as used for confocal Raman microscopy.

Raman measurements were performed with a WITec alpha 300 RA (WITec, Germany) confocal Raman microscope. The microscope was equipped with a $532 \mathrm{~nm}$ laser that was used at a power of $50 \mathrm{~mW}$. Spectra were acquired by a WITec UHTS 300 VIS-NIR spectrometer with a Peltier-cooled back-illuminated CCD-camera (1024 pixel) and a 1200 grooves $\mathrm{mm}^{-1}$ optical grating. The system had a spectral resolution of approximately $2 \mathrm{~cm}^{-1}$. Single spectra were acquired with an integration time of $5 \mathrm{~s}$ and by accumulating 5 spectra after $1 \mathrm{~min}$ of photo-bleaching. Depth scans through membranes were performed with equal settings, yet for reasons of time and sample stability without photo-bleaching prior to the acquisition of spectra. Omitting photobleaching did not affect EW trends over depth (see EW of NR-211 at beginning of test (BOT) in Figure 4c). Single spectra of the depth scans were acquired for $5 \mathrm{~s}$ and with 5 accumulations in $1 \mu \mathrm{m}$ intervals through hydrated membranes. For the membrane degradation analysis, 10 depth scans at different locations per sample were performed.

The axial resolution of the setup was $1.6 \mu \mathrm{m}$ (full width at half maximum intensity of the $520 \mathrm{~cm}^{-1}$ peak profile of $\mathrm{Si}$ ), determined by a confocal depth scan on a plain Si wafer. Since the refractive index of hydrated Nafion is almost identical to water $\left(1.35\right.$ and $\left.1.33,{ }^{41}\right)$, spherical aberration is virtually not existent for a microscope objective corrected for water immersion such as the Zeiss W Plan-Apochromat $63 \times / 1.0$ used in this work. Thus, axial focus shifts are assumed to be $<0.5 \mu \mathrm{m}$ in all thickness measurements of Nafion. ${ }^{42}$ Signal intensity losses by absorption or scattering with increasing focus depth were negligible in our data (Figure S4). For membrane thickness measurements based on depth scans the z-position was considered to be inside the membrane when the summarized spectral intensity within the range of 200 to $1500 \mathrm{~cm}^{-1}$ was equal or greater than $50 \%$ of the maximum signal intensity of that specific scan. In order to compare different depth scans through membranes, EW-results of individual scans of a sample were scaled with bilinear filtering to meet the mean membrane thickness of this sample. For the multi-layered membrane Nafion XL, the upper and lower membrane limits were chosen manually since the Raman intensity profile is not constant for this sample due to the PTFE-reinforcement with its different spectral area.

Constant heat input to a sample by long measurement times potentially leads to thermal degradation and thereby might alter the ratio between peaks in the Raman spectrum. By performing a time-series 


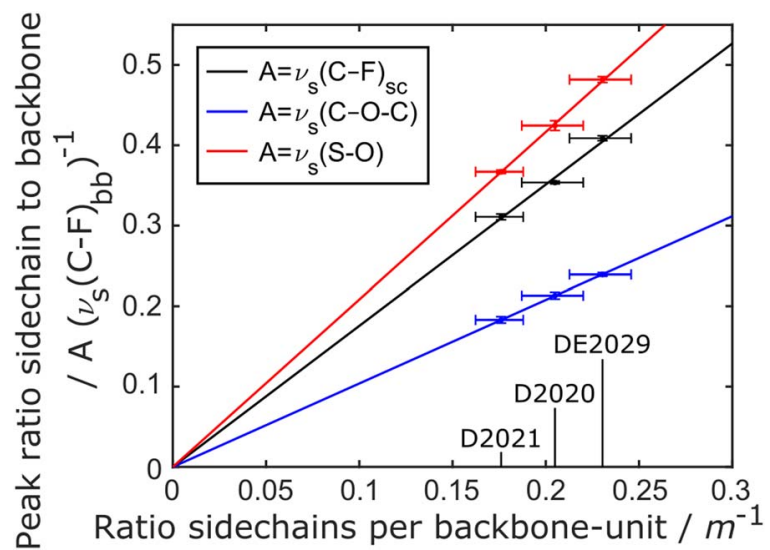

Figure 2. Correlation between Raman spectrum and sidechain to backbone repeat unit ratio of Nafion dispersions. Shown are mean ratios of different Raman sidechain peaks to the $v_{\mathrm{s}}(\mathrm{C}-\mathrm{F})_{\mathrm{bb}}$ peak over the ratio of sidechains per backbone repeat unit of the Nafion dispersions DE2029 ( $m=4.34)$, D2020 ( $m$ $=4.88)$, and D2021 ( $m=5.68)$. Horizontal error bars indicate EW tolerance as provided by the manufacturer and vertical error bars indicate the SD of the single Raman spectra that were acquired per sample.

over 30 min of continuous Raman measurements at a single location on a Nafion membrane, we excluded that the ionomer was degraded by our measurement settings (Figure S5).

Data processing.- - Removal of cosmic rays and background subtraction of the Raman spectra was performed using WITec Project FIVE+. Filter settings for cosmic ray removal in single spectra were chosen manually as required in order to remove artifacts. Datasets from confocal depth scans were automatically processed by a filter size of 4 and a dynamic factor of 5. Background subtraction was applied equally on all acquired data using the shape-based algorithm of the software with a shape size of 100 and a noise factor of 1 .

MATLAB (MathWorks, USA) was used for all further processing steps. First, a calibration curve for Nafion was established using the commercially available dispersions Nafion DE2029, Nafion D2020, and Nafion D2021. The area under the curve of the four vibrational modes $v_{\mathrm{s}}(\mathrm{C}-\mathrm{F})_{\mathrm{bb}}\left(685\right.$ to $\left.760 \mathrm{~cm}^{-1}\right), v_{\mathrm{s}}(\mathrm{C}-\mathrm{F})_{\mathrm{sc}}\left(770\right.$ to $\left.831 \mathrm{~cm}^{-1}\right), v_{\mathrm{s}}(\mathrm{C}-$ $\mathrm{O}-\mathrm{C})\left(945\right.$ to $\left.1001 \mathrm{~cm}^{-1}\right)$, and $\nu_{\mathrm{s}}(\mathrm{S}-\mathrm{O})\left(1037\right.$ to $\left.1081 \mathrm{~cm}^{-1}\right)$ was summarized. By plotting the ratios between the different sidechain-related peak intensities and the backbone-related peak intensity $v_{\mathrm{s}}(\mathrm{C}-\mathrm{F})_{\mathrm{bb}}$ over the inverse number of backbone repeat units per sulfonyl group $(1 / \mathrm{m}$, as provided by the manufacturer of the Nafion dispersions), a linear correlation between Raman peak intensity ratio and ionomer EW was obtained (Figure 2) according to Equation 1. Five single measurements were acquired at different locations per ionomer sample and were used as input for a linear regression. Under the premise that pure PTFE does not show any of the sidechain-related peaks, linear fits were created that meet the origin. The slope of these linear fits (Table I) was used to calculate the EW of all other investigated Nafion-samples according to their Raman spectra. The $95 \%$ confidence interval was calculated based on the 15 single data points that were used for the linear regression, and is provided for an EW of $1000 \mathrm{~g} \mathrm{~mol}^{-1}(m=5.56)$.

Table I. Slopes and coefficients of determination of the linear correlation between Raman intensity ratios and the ratio of sidechains per backbone repeat unit as displayed in Figure 2.

\begin{tabular}{lll}
$\mathrm{y}=\mathrm{A} / \nu_{\mathrm{s}}(\mathrm{C}-\mathrm{F})_{\mathrm{bb}}$ & Linear correlation & Coefficient of determination \\
\hline $\mathrm{A}=v_{\mathrm{s}}(\mathrm{C}-\mathrm{F})_{\mathrm{sc}}$ & $\mathrm{y}=1.7559 \cdot(1 / m)$ & $\mathrm{R}^{2}=0.9837$ \\
$\mathrm{~A}=v_{\mathrm{s}}(\mathrm{C}-\mathrm{O}-\mathrm{C})$ & $\mathrm{y}=1.0390 \cdot(1 / m)$ & $\mathrm{R}^{2}=0.9805$ \\
$\mathrm{~A}=v_{\mathrm{s}}(\mathrm{S}-\mathrm{O})$ & $\mathrm{y}=2.0828 \cdot(1 / m)$ & $\mathrm{R}^{2}=0.9920$
\end{tabular}

\section{Results and Discussion}

Raman spectrum of Nafion.-The Raman spectrum of Nafion (Figure 1b) has been extensively studied in literature, with most of the peaks being unambiguously assigned to modes of either the PTFEbackbone or the sidechain. ${ }^{26,41,43,44}$ In order to quantitatively measure the EW of an ionomer, strong peaks without overlapping neighboring peaks are preferable. The sharpest and most prominent peak of the backbone in Nafion is the symmetric stretching mode $v_{s}(\mathrm{C}-\mathrm{F})$ at $732 \mathrm{~cm}^{-1}$. Other backbone-associated modes like the peaks at around $290,385,1300$, and $1380 \mathrm{~cm}^{-1}$ are still observable in Nafion, yet they are broader than in $\mathrm{PTFE}^{26}$ and due to the superposition with adjacent peaks they are less suited for quantification (see Figure S6a for a direct comparison of the Raman spectra of Nafion and PTFE, and Figure 1a for the structure formula of Nafion).

Raman peaks of Nafion that have been assigned to sidechain-related vibrations are at 804,972 , and $1060 \mathrm{~cm}^{-1}$. The peak at $1060 \mathrm{~cm}^{-1}$ has been assigned to the $v_{\mathrm{s}}(\mathrm{S}-\mathrm{O})$ mode in very good accordance with the same vibrational mode as observed in methanesulfonate. ${ }^{45}$ While the peak at $972 \mathrm{~cm}^{-1}$ has been concordantly attributed to the ether bond symmetric stretch $\nu_{\mathrm{s}}(\mathrm{C}-\mathrm{O}-\mathrm{C})$ within the Nafion sidechain, ${ }^{26,41,43}$ literature is less consistent regarding the peak at $804 \mathrm{~cm}^{-1}$. Bribes et al. proposed the $v_{\mathrm{s}}(\mathrm{C}-\mathrm{S})$ mode to be responsible for this peak. ${ }^{43}$ However, after comparing the spectrum of the long side chain (LSC) ionomer Nafion with the short side chain (SSC) ionomer Aquivion, Peng et al. attributed this peak to the stretching mode of the $\mathrm{C}-\mathrm{F}$ bonds within the ionomer sidechain. ${ }^{41}$ A comparison of the spectra of LSC and SSC ionomers with a virtually identical ratio $m$ between backbone repeat units and sidechains (Figure 1c) reveals a strong difference in the intensity of the peak at $804 \mathrm{~cm}^{-1}$. Since both SSC and LSC ionomer only contain one sulfonyl group per sidechain, the intensity of this peak is expected to be approximately equal when contributing it to the $\nu_{\mathrm{s}}(\mathrm{C}-\mathrm{S})$ mode. Nafion, however, has a longer sidechain and therefore harbors more C-F bonds per sidechain than Aquivion (Figure 1a). We therefore agree with the work by Peng et al. and suggest the assignment of the C-F stretch of the sidechain $v(C-F)_{s c}$ to the peak at $804 \mathrm{~cm}^{-1}, 41$ while the same vibrational mode of the ionomer backbone $v_{\mathrm{s}}(\mathrm{C}-\mathrm{F})_{\mathrm{bb}}$ is located at $732 \mathrm{~cm}^{-1}$.

EW-Raman intensity calibration curve.-Raman spectra are often analyzed only qualitatively since the absolute intensity of a sample not only depends on the sample, but, for example, also on the sensitivity of the spectrometer and on measurement settings like laser power and integration time. Therefore, a reliable normalization method is required to compare spectra quantitatively. Korzeniewski et al. established confocal Raman microscopy as a tool for quantifying the EW of bis-perfluorosulfonyl imide ionomers by normalizing spectra relative to their total spectral area. ${ }^{33}$ This normalization method is suitable for compensating spectral data of samples with different densities. However, it is a potential source of bias when analyzing ionomers with Raman spectra that are substantially different from the spectrum of the original polymer, because this normalization approach is performed under the assumption that the total spectral intensity of the ionomer remains constant when changing the ratio $m$ of backbone repeat units per sidechain (Figure 1a). Thus, we used a quantification approach for the EW of Nafion that is independent from this normalization-related bias. As the EW of an ionomer is determined by the ratio between sidechains and backbone repeat units, one can focus on the ratio between two Raman peaks that are related to sidechain and backbone in order to calculate the EW without the need to normalize the data. As the intensity of a Raman peak is directly dependent on the number of sampled molecules, ${ }^{46}$ the following correlation can be established:

$$
\frac{1}{m}=\frac{K \cdot n \cdot a_{s c} \cdot I_{s c}}{K \cdot n \cdot a_{b b} \cdot I_{b b}}=\frac{a_{s c}}{a_{b b}} \cdot \frac{I_{s c}}{I_{b b}}=a \cdot \frac{I_{s c}}{I_{b b}}
$$

with the number of backbone repeat units per sidechain $m$, Raman measurement constant $K$ (e.g. excitation wavelength and laser power), the number of sampled molecules $n$, and the Raman intensities of a sidechain-related peak $I_{s c}$ and a backbone-related peak $I_{b b}$. The two 


\section{Table II. EW values of commercial Nafion membranes measured with confocal Raman microscopy. Five measurements were performed per sample and the mean result is provided.}

\begin{tabular}{lll} 
Sample & $\begin{array}{l}\text { EW, manufacturer } \\
{\left[\mathrm{g} \mathrm{mol}^{-1}\right]}\end{array}$ & $\begin{array}{l}\text { EW } \pm \mathrm{SD}, \\
\text { measured }\left[\mathrm{g} \mathrm{mol}^{-1}\right]\end{array}$ \\
\hline Nafion N117 & $990-1053$ & $1028 \pm 6$ \\
Nafion NR-211 & $990-1053$ & $1027 \pm 3$ \\
Nafion NR-212 & $990-1053$ & $1022 \pm 6$
\end{tabular}

factors $a_{s c}$ and $a_{b b}$ represent the specific Raman activities of the two investigated vibrational modes. All spectral information is obtained within one single measurement and therefore $n$ and $K$ are identical for both investigated peaks. The specific Raman activity of the peaks is unknown. Consequently, the factor $\mathrm{a}_{\mathrm{sc}} / \mathrm{a}_{\mathrm{bb}}$ must be determined experimentally for every pair of investigated sidechain- and backbone-peaks, leading to a simplified version of the equation with $a$ being the slope of a linear correlation. Equation 1 also holds true for PTFE with no sidechain-peaks (Figure S6a) and therefore meets the origin at infinite $m$. In order to establish this correlation as a calibration curve for Nafion, we measured different Nafion samples with known EW (DE2029, D2020, and D2021). Figure 2 shows the intensity ratio of several sidechain-related vibrational modes $\left(v_{\mathrm{s}}(\mathrm{C}-\mathrm{F})_{\mathrm{sc}}, v_{\mathrm{s}}(\mathrm{C}-\mathrm{O}-\mathrm{C})\right.$, or $\left.\nu_{\mathrm{s}}(\mathrm{S}-\mathrm{O})\right)$ divided by the backbone-associated $\nu_{\mathrm{s}}(\mathrm{C}-\mathrm{F})_{\mathrm{bb}}$ mode over the sidechain to backbone repeat unit ratio $1 / \mathrm{m}$. For all the investigated sidechain peaks, a linear correlation exists (Table I), with only the slope being different as expected.

Although displaying the ratio of Raman peaks directly over the EW of the ionomer seems more intuitive, there is no linear correlation between both, since the EW is not a ratio between sidechains and backbone repeat units, but a ratio between the total weight of the ionomer (sidechains and backbone) and the sidechains. Also, it is noteworthy that from the structure formula of the ionomer (Figure 1a) one would expect $1 /(m+1)$ to be the correct estimate for the Raman peak intensity ratio in Equation 1, since basically one backbone repeat unit is considered as part of the ionomer sidechain. However, we found empirically that using $1 / m$ instead of $1 /(m+1)$ in Equation 1 results in a better linear correlation. This finding can be explained by a potential influence of the ether-bond that connects sidechain and backbone on the wavenumber of the $\nu_{\mathrm{s}}(\mathrm{C}-\mathrm{F})$ mode of directly adjacent $\mathrm{C}-\mathrm{F}$ bonds in the polymer main chain (Figure 1a). This hypothesis is consistent with the distinction between the vibrational modes $v_{\mathrm{s}}(\mathrm{C}-\mathrm{F})_{\mathrm{sc}}$ and $\nu_{\mathrm{s}}(\mathrm{C}-\mathrm{F})_{\mathrm{bb}}$, which is also based on a different Raman shift of the symmetric $\mathrm{C}-\mathrm{F}$ stretch in the ionomer sidechain as compared to the same vibrational mode in the backbone.

The symmetric stretch mode of the sulfonyl group shows the highest intensity of all investigated sidechain-peaks (Figure 1) and consequently the impact of random spectral noise on the result is lowest when using this peak for the analysis. Also, the sulfonyl group is the best indicator for the EW of an ionomer when taking into account that partial degradation of sidechains might leave one or both of the ether-bonds in place but remove the sulfonyl group. ${ }^{10}$ Therefore, we used the calibration curve between the $\nu_{\mathrm{s}}(\mathrm{S}-\mathrm{O})$ mode and the $\nu_{\mathrm{s}}(\mathrm{C}-\mathrm{F})_{\mathrm{bb}}$ mode for all further measurements. Note that all measurements were performed in the hydrated form of the ionomers in order to achieve equal intensities of the hydration-dependent $\nu_{\mathrm{s}}(\mathrm{S}-\mathrm{O})$ mode (compare Figure S3a).

EW-measurement of commercial Nafion membranes.-For Nafion N117, NR-211, and NR-212 the manufacturer states an EW range from 990 to $1053 \mathrm{~g} \mathrm{~mol}^{-1}$. Using confocal Raman microscopy we obtained values between 1022 and $1028 \mathrm{~g} \mathrm{~mol}^{-1}$ for the three membranes (Table II). According to the manufacturer only casting procedure (extrusion or dispersion-casting) and thickness of the membranes differ. Thus, a highly similar if not identical EW can be expected for the membranes as they are based on the same, chemically stabilized form of Nafion. While the EW of extruded Nafion N11x membranes is equal to the EW of dispersion-cast NR-21x membranes, the crystallinity of the two membrane types was reported to be different (see ${ }^{2}$ and sources therein). Raman spectroscopy is sensitive toward crystallinity changes in PTFE, ${ }^{47}$ and Gruger et al. suggested a change in the width of the $\nu_{\mathrm{s}}(\mathrm{C}-\mathrm{F})_{\mathrm{bb}}$ mode according to the sample crystallinity. ${ }^{26}$ Despite these reports no observable differences exist in our data between extruded and dispersion-cast Nafion (Figure S6b). We therefore conclude that at least within the crystallinity range of commercially available Nafion membranes the differences are too small to have a significant impact on the investigated Raman peaks.

Moukheiber et al. published a comprehensive analysis of the accuracy of different chemical methods that are available for the determination of an ionomer's EW. ${ }^{25}$ Methods such as titration, EDX, elemental analysis, and IR spectroscopy were used to determine the EW of various ionomers and the accuracy of the measurements was presented as a deviation from the values given by the manufacturer. These methods typically featured standard deviations (SD) from $\pm 0.05 \mathrm{meq} \mathrm{g}^{-1}$ (ion exchange capacity) up to $\pm 0.08 \mathrm{meq}^{-1}$. This corresponds roughly to $\pm 50 \mathrm{~g} \mathrm{~mol}^{-1}$ up to $\pm 80 \mathrm{~g} \mathrm{~mol}^{-1}$ for a Nafion membrane with an EW of $1000 \mathrm{~g} \mathrm{~mol}^{-1}$. In contrast, the confocal Raman microscopy approach proved to be more accurate with a $95 \%$ confidence interval ( $\pm 2 \mathrm{SD}$ ) of $\pm 13 \mathrm{~g} \mathrm{~mol}^{-1}$ for an $\mathrm{EW}=1000 \mathrm{~g} \mathrm{~mol}^{-1}$ Nafion sample according to our calibration curve (Figure 2).

Next, we applied our approach on the multi-layered membrane Nafion XL. Since it is not reasonable to determine the EW of this reinforced membrane based on single-spot measurements, as there are substantial differences between the outer Nafion layers and the inner PTFE-reinforcement, a confocal depth scan through the membrane was performed. Figure 3a depicts the calculated EW of Nafion XL over membrane thickness. The outer layers (pure Nafion with $\mathrm{SiO}_{2}$ particles) ${ }^{48}$ of Nafion XL show a highly homogeneous EW of around $970 \mathrm{~g} \mathrm{~mol}^{-1}$ while within the microporous PTFE reinforcement layer the EW is less homogeneous and reaches peaks of more than $2000 \mathrm{~g}$ $\mathrm{mol}^{-1}$. A clear distinction between PTFE and Nafion within the reinforcement layer is not possible due to the resolution limits of confocal light microscopy. Thus, the EW trend of the depth scan creates the impression of local EW changes, which actually originate from local distribution differences between Nafion and PTFE-reinforcement that are beyond the spatial resolution of confocal light microscopy. Although Nafion that is present in the pores of the reinforcement layer is similar to the Nafion in the outer layers, the increasing ratio of PTFE to Nafion in the reinforcement layer results in very high EWs around $2000 \mathrm{~g} \mathrm{~mol}^{-1}$. A virtual cross-section of Nafion XL is shown in Figure $3 b$, with the different chemical phases displayed in Figure $3 \mathrm{c}$, which highlights the inhomogeneous reinforcement layer of the membrane. The EW results of Nafion XL are roughly consistent with the values provided by Moukheiber et al., who determined the EW of Nafion XL with EDX to approx. $910 \mathrm{~g} \mathrm{~mol}^{-1}$ in the Nafion-rich outer layers and approx. $1790 \mathrm{~g} \mathrm{~mol}^{-1}$ in the PTFE-rich central layer. ${ }^{25}$ A possible explanation for the lower EW in the outer layers of Nafion XL determined by Moukheiber et al. as compared to this work is the potential influence of the silica particles in Nafion $\mathrm{XL}^{48}$ on EDX-measurements, which may add additional oxygen-signals and therefore skew the result toward a higher sidechain-content.

Membrane degradation analysis._-Besides analyzing standard and reinforced membranes, confocal Raman microscopy can also be employed to investigate ionomer membranes after operation in a PEMFC. For this purpose, a Nafion NR-211 membrane was operated in accelerated stress test conditions according to DOE test for chemical membrane degradation (here: $100 \mathrm{~h}$ operation at OCV conditions). Within this timeframe, the cell voltage dropped remarkably from $0.94 \mathrm{~V}$ to $0.21 \mathrm{~V}$, with a strong decrease in the first $10 \mathrm{~h}$, followed by a plateau and another strong decrease after $75 \mathrm{~h}$ (Figure 4a). This trend including the spikes from interrupting the OCV hold is consistent with published data ${ }^{49}$ and most probably reflects membrane thinning and pinhole formation, leading to increased gas permeation and radical formation, which in turn attack the membrane polymer. $\mathrm{H}_{2}$ crossover supports this interpretation, showing a more than 
a

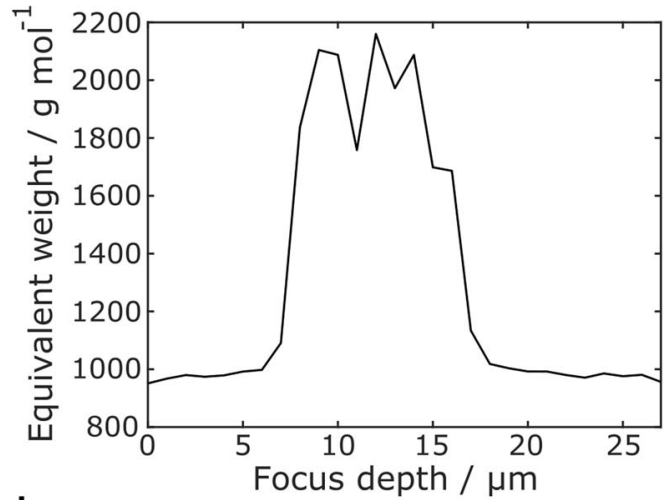

b

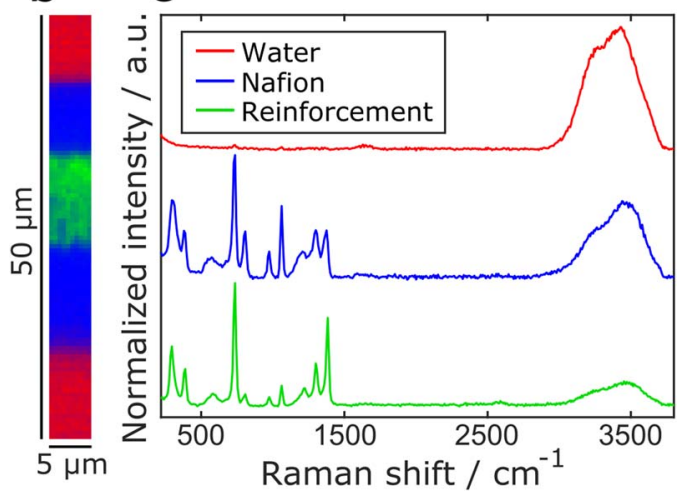

Figure 3. Characterization of Nafion XL with confocal Raman microscopy. a) Single depth scan through Nafion XL. Raman spectra were taken at every $1 \mu \mathrm{m}$ and the EW over depth is displayed. b) Virtual cross-section through water-immersed Nafion XL with false-color display of the different phases in the membrane. c) Raman spectra of the chemical phases in Nafion XL. The scan was performed with a 300 grooves $\mathrm{mm}^{-1}$ optical grating, at an isotropic pixel size of $0.5 \mu \mathrm{m}$, and with an integration time of $0.5 \mathrm{~s}$ per pixel. Spectra are normalized to maximum peak intensity. Phase assignment in the Raman image was performed using a custom-built MATLAB code.

tenfold increase from $4.35 \mathrm{~mA} \mathrm{~cm}^{-2}$ at BOT to $68.43 \mathrm{~mA} \mathrm{~cm}^{-2}$ after $100 \mathrm{~h}$ OCV hold, with a strong increase after $75 \mathrm{~h}$ (Figure $4 \mathrm{~b}$ ).

Using confocal Raman microscopy, we determined the thickness of the membranes after electrode removal. The thickness of the hydrated membrane decreased from $30.1 \mu \mathrm{m}$ (BOT) to $11.0 \mu \mathrm{m}$ after $100 \mathrm{~h}$ OCV (Figure 4b, Table III). Thickness discrepancy of NR-211 at BOT from the nominal value given by the manufacturer $(25.4 \mu \mathrm{m})$ is attributed to membrane swelling, since measurements were performed in water immersion. Note that the reported thinning corresponds to the measured average thickness only. Thus, small deviations such as the formation of local pinholes were not taken into account. Between BOT and $75 \mathrm{~h} \mathrm{OCV}$, a correlation between membrane thinning and increasing $\mathrm{H}_{2}$ crossover can be seen (Figure $4 \mathrm{~b}$ ), which can be explained by

Table III. Fuel cell and membrane parameters measured prior and post accelerated stress testing. EW and membrane thickness are given as mean values \pm SD of 10 individual measurements.

\begin{tabular}{llll} 
& $\begin{array}{l}\text { Mean EW } \\
{\left[\mathrm{g} \mathrm{mol}^{-1}\right]}\end{array}$ & $\begin{array}{l}\text { Membrane } \\
\text { thickness }[\mu \mathrm{m}]\end{array}$ & $\begin{array}{l}\mathrm{H}_{2} \text {-crossover } \\
{\left[\mathrm{mA} \mathrm{cm}^{-2}\right]}\end{array}$ \\
\hline BOT & $1035 \pm 10$ & $30.1 \pm 0.3$ & 4.35 \\
25 h OCV & $1076 \pm 30$ & $28.8 \pm 2.9$ & 5.64 \\
50 h OCV & $1107 \pm 67$ & $19.9 \pm 2.6$ & 6.29 \\
$75 \mathrm{~h} \mathrm{OCV}$ & $1171 \pm 101$ & $16.6 \pm 3.4$ & 13.46 \\
100 h OCV & $1200 \pm 127$ & $11.0 \pm 3.1$ & 68.43
\end{tabular}
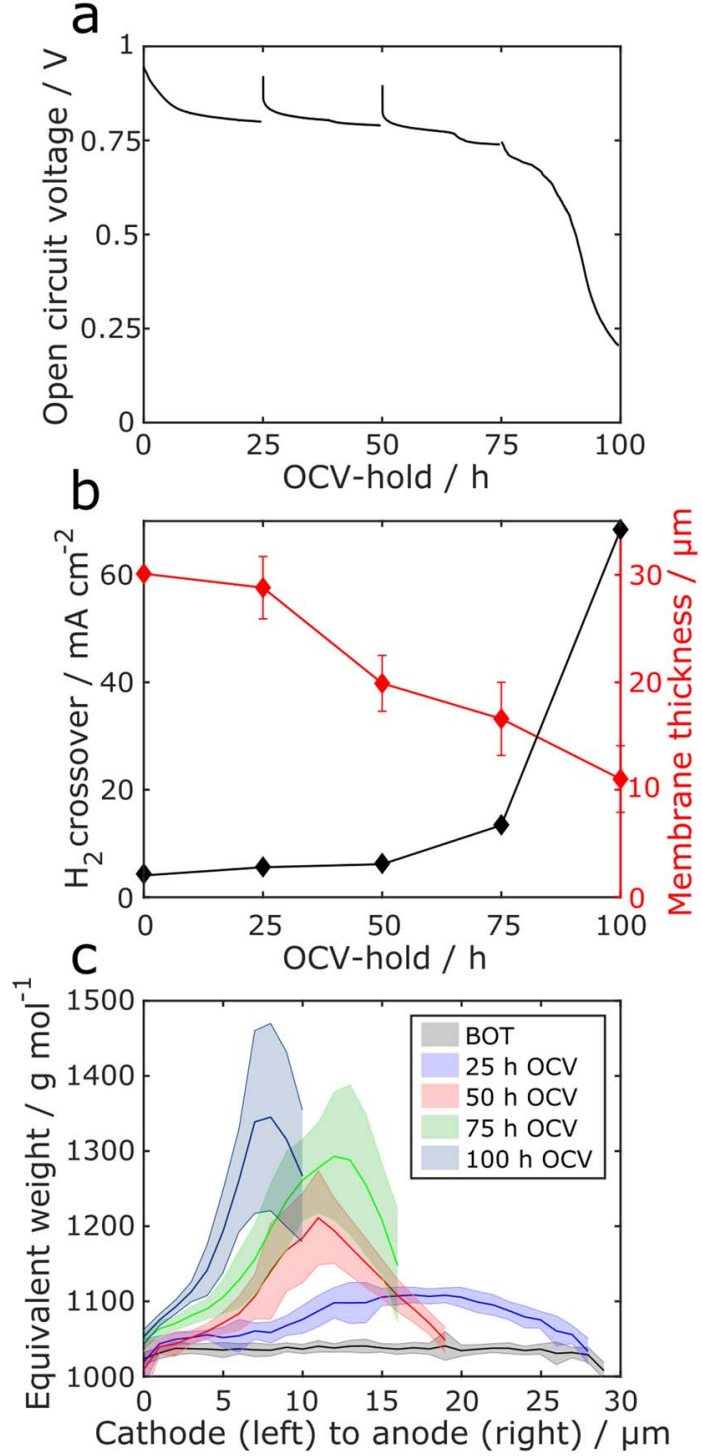

Figure 4. Membrane changes upon accelerated stress testing. a) OCV over time. b) Gas crossover and membrane thickness over time. $\mathrm{H}_{2}$ crossover was taken from LSV at $0.2 \mathrm{~V}$ (Figure S2a). Membrane thickness values are mean thicknesses $\pm \mathrm{SD}$ of 10 confocal depth scans per sample. c) EW changes during OCV hold over membrane z-position. Lines correspond to mean of 10 measurements $\pm \mathrm{SD}$

an increase in the gas diffusion rate with decreasing membrane thickness. The steep increase in $\mathrm{H}_{2}$ crossover from $75 \mathrm{~h}$ to $100 \mathrm{~h} \mathrm{OCV}$ hold most likely reflects the formation of pinholes in the membrane, which increase gas crossover substantially and independently from the measured thickness.

The EW of aged membranes was determined by applying the calibration curve obtained in Figure 2 on confocal depth scans of aged Nafion NR-211. With this approach the EW of the membranes was quantified locally between cathode and anode using the high spatial resolution of confocal light microscopy in the low single-digit micrometer range. We found that the $\mathrm{EW}$ increased gradually with longer $\mathrm{OCV}$ times, with only a minor effect on the cathode side and the highest increase between the anode and the center of the membrane (Figure 4c). Since Ghassemzadeh and Holdcroft found that for NR-211 chemical degradation occurs mainly via cleavage of sidechains, ${ }^{10} \mathrm{EW}$ measurements are a valid indicator for membrane ageing. Our results support the theory of Liu and Zuckerbrod, who found a higher concentration of $\mathrm{H}_{2} \mathrm{O}_{2}$ at the anode side of a PEMFC, ${ }^{12}$ which may lead to a 
preferred radical formation at the catalyst layer of the anode side and subsequently lead to an anisotropically accelerated membrane degradation at the anode of the fuel cell. Similar results have been reported by Zatoń et al., who performed Raman spectroscopy on Nafion membranes of aged MEAs and found that sidechain-related peaks were lost mostly on the anode side. ${ }^{30}$ However, in our data we see the most pronounced increase in the EW not directly at the anode side, but between the anode and the center of the membrane. Based on our results, we hypothesize for the entire process of membrane thinning that membrane degradation initially increases the EW by the loss of sidechains. This might be followed by breakdown of the PTFE-backbone, which is reflected in the decrease of the EW toward the anode (Figure 4c). Finally, the ionomer is completely lost, which reduces overall membrane thickness.

Besides an anisotropic ageing between anode and cathode our data also shows that degradation proceeds inhomogeneously over the xyplane of the membrane. As it can be seen in Figure 4c and Table III, the SD of the single EW measurements increased with longer OCV holds, meaning that local discrepancies in the EW of the membrane became larger with more elevated degradation status. Inhomogeneous membrane ageing is consistent with the work of Shi and Baker, who analyzed the spatial distribution of signs of chemical degradation in a Nafion NR-212 membrane. ${ }^{11}$

An OCV hold as an accelerated stress test shows rapid membrane degradation, but the underlying ageing does not necessarily display the same progression as in fuel cells operated at normal working conditions. Also, changes in fuel cell design such as electrode morphology and catalyst loading may influence ageing mechanisms. Thus, different cell types and working conditions are a plausible explanation for the contradictory results found in literature that suggest membrane degradation to take place preferentially on the anode side ${ }^{16-18}$ or the cathode side ${ }^{15,19-21}$ of a MEA. Further, Chandesris et al. found that not all experimental approaches that have been used so far might be suitable for a reliable spatial analysis of membrane degradation. ${ }^{18}$ Confocal Raman microscopy as a post mortem analysis tool to investigate the degradation of ionomer membranes can provide valuable data of the spatial distribution of ageing. In future, this method can complement established techniques in order to elucidate degradation mechanisms and factors that influence membrane ageing.

\section{Conclusions}

In this work we investigated the capabilities of confocal Raman microscopy for the analysis of ionomers that are used as proton exchange membranes in fuel cells. We developed a novel approach to use confocal Raman microscopy as a simple and non-destructive tool to quantify the EW of Nafion. Highly standardized measurement settings are required and a device-specific calibration curve has to be prepared in order to calculate the EW of an ionomer sample reliably. Our approach reaches a significantly higher accuracy than other published chemical methods, with a $95 \%$ confidence interval of $\pm 13 \mathrm{~g} \mathrm{~mol}^{-1}$ at an EW of $1000 \mathrm{~g} \mathrm{~mol}^{-1}$.

Further, confocal Raman microscopy offers the power of a depthresolved analysis of multi-layered and aged ionomer membranes. To demonstrate the spatial resolution of confocal Raman microscopy, we used our novel measurement method to investigate the applicationrelevant issue of membrane degradation. We evaluated the degradation of a Nafion NR-211 membrane within a PEMFC. Our results show that upon an OCV hold, as an accelerated stress test, the ionomer membrane exhibited substantial thinning which was accompanied by a clear increase in the EW. Both are signs of chemical-mechanical membrane degradation ${ }^{10,50}$ and their onsets could be observed already after short timespans of the employed stress test protocol. Electrochemical characterization of the PEMFC confirmed signs of ageing in agreement with results obtained by confocal Raman microscopy. While the pristine membrane showed a homogeneous EW distribution, the analysis of aged samples with confocal Raman microscopy revealed increasing differences in the xy-plane as well as along the $\mathrm{z}$ axis. Most pronounced EW increases of the membrane were observed closer to the anode side than the cathode side of the membrane. The fact that the highest EW values were found close to but not directly at the anode was attributed to the degradation mechanism, which is supposed to remove sidechains of the ionomer first, followed by the decomposition of the polymer backbone.

In summary, confocal Raman microscopy is a promising tool for quantitative ionomer characterization. It offers applications as a quality control for pristine membranes and as a quantitative analysis tool to obtain 3D information of degraded membranes. Ion exchange membrane characterization options with confocal Raman microscopy are manifold and are not limited to Nafion and fuel cells, but also include other PFSAs and different ionomer types for applications such as electrolysis or redox-flow batteries.

\section{Acknowledgments}

The authors thank Dr. Timo Kurz for providing access to the laser engraver and help with electrode ablation of MEAs, and Brian Shanahan for proof-reading of the manuscript. The authors gratefully acknowledge financial support of this work by the Federal Ministry of Education and Research in Germany within the project DEKADE (grant number: 03SF0544D).

\section{ORCID}

Simon Thiele (1D https://orcid.org/0000-0002-4248-2752

Severin Vierrath (1) https://orcid.org/0000-0002-4505-2803

\section{References}

1. Y. Wang, K. S. Chen, J. Mishler, S. C. Cho, and X. C. Adroher, Appl. Energy, 88(4), 981 (2011).

2. A. Kusoglu and A. Z. Weber, Chem. Rev., 117(3), 987 (2017).

3. L. Dubau, L. Castanheira, F. Maillard, M. Chatenet, O. Lottin, G. Maranzana, J. Dillet, A. Lamibrac, J.-C. Perrin, E. Moukheiber, A. ElKaddouri, G. de Moor, C. Bas, L. Flandin, and N. Caqué, WIREs Energy Environ., 3(6), 540 (2014).

4. J. Wu, X. Z. Yuan, J. J. Martin, H. Wang, J. Zhang, J. Shen, S. Wu, and W. Merida, J. Power Sources, 184(1), 104 (2008).

5. T. Jahnke, G. Futter, A. Latz, T. Malkow, G. Papakonstantinou, G. Tsotridis, P. Schott, M. Gérard, M. Quinaud, M. Quiroga, A. A. Franco, K. Malek, F. Calle-Vallejo, R. Ferreira de Morais, T. Kerber, P. Sautet, D. Loffreda, S. Strahl, M. Serra, P. Polverino, C. Pianese, M. Mayur, W. G. Bessler, and C. Kompis, J. Power Sources, 304, 207 (2016).

6. M. Feng, R. Qu, Z. Wei, L. Wang, P. Sun, and Z. Wang, Sci. Rep., 5, 9859 (2015).

7. S. J. Osborn, M. K. Hassan, G. M. Divoux, D. W. Rhoades, K. A. Mauritz, and R. B. Moore, Macromolecules, 40(10), 3886 (2007)

8. J. Zhang, Z. Xie, J. Zhang, Y. Tang, C. Song, T. Navessin, Z. Shi, D. Song, H. Wang, D. P. Wilkinson, Z.-S. Liu, and S. Holdcroft, J. Power Sources, 160(2), 872 (2006).

9. E. Endoh, S. Terazono, H. Widjaja, and Y. Takimoto, Electrochem. Solid-State Lett., 7(7), A209 (2004).

10. L. Ghassemzadeh and S. Holdcroft, J. Am. Chem. Soc., 135(22), 8181 (2013).

11. W. Shi and L. A. Baker, RSC Adv., 5(120), 99284 (2015).

12. W. Liu and D. Zuckerbrod, J. Electrochem. Soc., 152(6), A1165 (2005)

13. L. Gubler, S. M. Dockheer, and W. H. Koppenol, J. Electrochem. Soc., 158(7), B755 (2011).

14. T. Kinumoto, M. Inaba, Y. Nakayama, K. Ogata, R. Umebayashi, A. Tasaka, Y. Iriyama, T. Abe, and Z. Ogumi, J. Power Sources, 158(2), 1222 (2006).

15. A. Pozio, R. F. Silva, M. de Francesco, and L. GIORGI, Electrochim. Acta, 48(11), 1543 (2003).

16. N. Ohguri, A. Y. Nosaka, and Y. Nosaka, J. Power Sources, 195(15), 4647 (2010).

17. C. Huang, K. Seng Tan, J. Lin, and K. Lee Tan, Chem. Phys. Lett., 371(1-2), 80 (2003).

18. M. Chandesris, R. Vincent, L. Guetaz, J.-S. Roch, D. Thoby, and M. Quinaud, Int. J. Hydrogen Energy, 42(12), 8139 (2017).

19. H. Ericson, T. Kallio, T. Lehtinen, B. Mattsson, G. Sundholm, F. Sundholm, and P. Jacobsson, J. Electrochem. Soc., 149(2), A206 (2002).

20. S. Kundu, M. W. Fowler, L. C. Simon, R. Abouatallah, and N. Beydokhti, J. Power Sources, 183(2), 619 (2008).

21. L. Zhang and S. Mukerjee, J. Electrochem. Soc., 153(6), A1062 (2006).

22. D. E. Curtin, R. D. Lousenberg, T. J. Henry, P. C. Tangeman, and M. E. Tisack, J. Power Sources, 131(1-2), 41 (2004).

23. K. D. Kreuer, M. Schuster, B. Obliers, O. Diat, U. Traub, A. Fuchs, U. Klock, S. J. Paddison, and J. Maier, J. Power Sources, 178(2), 499 (2008).

24. T.-Y. Chen and J. Leddy, Langmuir, 16(6), 2866 (2000).

25. E. Moukheiber, G. de Moor, L. Flandin, and C. Bas, J. Memb. Sci., 389, 294 (2012).

26. Alain Gruger, André Régis, Tatiana Schmatko, and Philippe Colomban, Vib. Spectrosc., 26, 215 (2001). 
27. Y. Tabuchi, R. Ito, S. Tsushima, and S. Hirai, J. Power Sources, 196(2), 652 (2011)

28. M. Falk, Can. J. Chem., 58, 1495 (1980).

29. H. Matic, A. Lundblad, G. Lindbergh, and P. Jacobsson, Electrochem. Solid-State Lett., 8(1), A5 (2005).

30. M. Zatoń, J. Rozière, and D. J. Jones, J. Mater. Chem. A, 5(11), 5390 (2017).

31. S. A. Perusich, J. Appl. Polym. Sci., 120(1), 165 (2011).

32. E. Smith and G. Dent, Modern Raman Spectroscopy: A practical approach, Chichester, Wiley (2008).

33. C. Korzeniewski, Y. Liang, P. Zhang, I. Sharif, J. P. Kitt, J. M. Harris, S. J. Hamrock, S. E. Creager, and D. D. DesMarteau, Appl. Spectrosc., 72(1), 141 (2018).

34. Office of Energy Efficiency \& Renewable Energy, Fuel Cell Technologies Office Multi-Year Research, Development, and Demonstration Plan, https://energy.gov/eere/fuelcells/downloads/fuel-cell-technologies-office-multiyear-research-development-and-22, accessed: October, 2018

35. S. Radice, C. Oldani, L. Merlo, and M. Rocchia, Polym. Degrad. Stab., 98(6), 1138 (2013).

36. S. R. Lowry and K. A. Mauritz, J. Am. Chem. Soc., 102(14), 4665 (1980).

37. R. Buzzoni, S. Bordiga, G. Ricchiardi, G. Spoto, and A. Zecchina, J. Phys. Chem., 99(31), 11937 (1995).

38. G. Alberti, R. Narducci, and M. Sganappa, J. Power Sources, 178(2), 575 (2008).
39. Perma pure, Nafion: Physical and Chemical Properties, https://www.permapure.com/ products/nafion-tubing/nafion-physical-and-chemical-properties/, accessed: October, 2018.

40. J. Zięba-Palus and A. Michalska, Vib. Spectrosc., 74, 6 (2014)

41. Z. Peng, P. Huguet, S. Deabate, A. Morin, and A. K. Sutor, J. Raman Spectrosc., 44(2), 321 (2013).

42. S. H. Wiersma, P. Török, T. D. Visser, and P. Varga, J. Opt. Soc. Am. A, 14(7), 1482 (1997).

43. J.-L. Bribes, Boukari M. El, and J. Maillols, J. Raman Spectrosc., 22, 275 (1991).

44. N. Chourdakis and G. A. Voyiatzis, J. Polym. Sci. B Polym. Phys., 45(17), 2509 (2007).

45. M. G. Miles, G. Doyle, R. P. Cooney, and R. S. Tobias, Spectrochim. Acta A Mol. Biomol. Spectrosc., 25A, 1515 (1969).

46. G. Turrell and J. Corset, Raman Microscopy: Development and Applications, Amsterdam, Elsevier Acad. Press; Academic press (1996 // 2003).

47. J. F. Rabolt, J. Polym. Sci. Polym. Phys. Ed., 21(9), 1797 (1983).

48. S. Shi, A. Z. Weber, and A. Kusoglu, J. Memb. Sci., 516, 123 (2016).

49. M. Adamski, T. J. G. Skalski, B. Britton, T. J. Peckham, L. Metzler, and S. Holdcroft, Angew. Chem., 129 // 56(31), 9186 (2017).

50. C. Lim, L. Ghassemzadeh, F. van Hove, M. Lauritzen, J. Kolodziej, G. G. Wang, S. Holdcroft, and E. Kjeang, J. Power Sources, 257, 102 (2014). 